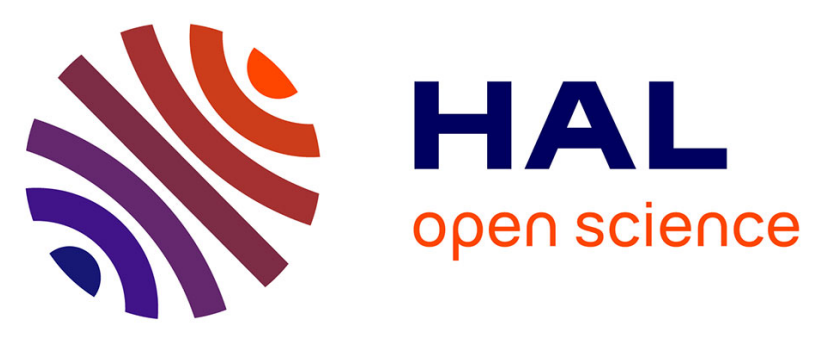

\title{
Redistribution of PDGFRbeta cells and NG2DsRed pericytes at the cerebrovasculature after status epilepticus
}

\author{
Sebastien Milesi, Badreddine Boussadia, Clement Plaud, Matthias Catteau, \\ Marie-Claude Rousset, Frédéric de Bock, Marie Schaeffer, Mireille \\ Lerner-Natoli, V. Rigau, Nicola Marchi
}

\section{To cite this version:}

Sebastien Milesi, Badreddine Boussadia, Clement Plaud, Matthias Catteau, Marie-Claude Rousset, et al.. Redistribution of PDGFRbeta cells and NG2DsRed pericytes at the cerebrovasculature after status epilepticus. Neurobiology of Disease, 2014, 71, pp.151-158. 10.1016/j.nbd.2014.07.010 . hal01944930

\author{
HAL Id: hal-01944930 \\ https://hal.science/hal-01944930
}

Submitted on 5 Dec 2018

HAL is a multi-disciplinary open access archive for the deposit and dissemination of scientific research documents, whether they are published or not. The documents may come from teaching and research institutions in France or abroad, or from public or private research centers.
L'archive ouverte pluridisciplinaire HAL, est destinée au dépôt et à la diffusion de documents scientifiques de niveau recherche, publiés ou non, émanant des établissements d'enseignement et de recherche français ou étrangers, des laboratoires publics ou privés. 
Neurobiol Dis. 2014 November ; 0: 151-158. doi:10.1016/j.nbd.2014.07.010.

\title{
Redistribution of PDGFR $\beta$ cells and NG2DsRed pericytes at the cerebrovasculature after status epilepticus
}

\author{
Sebastien Milesi ${ }^{1}$, Badreddine Boussadia ${ }^{1}$, Clement Plaud ${ }^{1}$, Matthias Catteau ${ }^{1}$, Marie- \\ Claude Rousset ${ }^{1}$, Frederic De Bock ${ }^{1}$, Marie Scheffer ${ }^{2}$, Mireille Lerner-Natoli ${ }^{1}$, Valerie \\ Rigau $^{3}$, and Nicola Marchi ${ }^{1,3}$ \\ ${ }^{1}$ Laboratory of Cerebrovascular Mechanisms of Brain Disorders, Department of Neuroscience, \\ Institute of Functional Genomics, Montpellier, France (Centre National Recherche Scientifique - \\ CNRS) \\ 2Laboratory of Networks and Rhythms in Endocrine Glands, Department of Physiology. Institute \\ of Functional Genomics, Montpellier, France (Centre National Recherche Scientifique - CNRS) \\ ${ }^{3}$ University Hospital, Pathology Department, Montpellier France
}

\section{Abstract}

Purpose-The role of cerebrovascular dysfunction in seizure disorders is recognized. Bloodbrain barrier (BBB) damage in epilepsy has been linked to endothelial and glial pathophysiological changes. Little is known about the involvement of pericytes, a cell type that contributes to BBB function.

\begin{abstract}
Methods-NG2DsRed mice were used to visualize cerebrovascular pericytes. The pattern of vascular and parenchymal distribution of platelet-derived growth factor receptor beta (PDGFR $\beta$ ) cells was evaluated by immunohistochemistry. Status epilepticus was induced in NG2DsRed or C57BL/6J mice by intraperitoneal kainic acid (KA). Animals were perfused intracardially using FITC-Dextran or FITC-Albumin to visualize the cerebrovasculature. Colocalization was performed between NG2DsRed, PDGFR $\beta$ and microglia IBA-1. Confocal 3D vessel reconstruction was used to visualize changes in cell morphology and position. PDGFR $\beta$ expression was also evaluated in vitro using organotypic hippocampal cultures $(\mathrm{OHC})$ treated with kainic acid to induce seizure-like activity. Co-localization of PDGFR $\beta$ with the vascular marker RECA- 1 and NG2 was performed. Finally, we assessed the expression of PDGFR $\beta$ in brain specimens obtained from a cohort of patients affected by drug resistant epilepsy compared to available autoptic brain.
\end{abstract}

(O2014 Elsevier Inc. All rights reserved.

Corresponding Author: Dr. Nicola Marchi. Cerebrovascular Mechanisms of Brain Disorders, Institut de Génomique Fonctionnelle (CNRS UMR5203, INSERM U661) 141 rue de la Cardonille, 34094 Montpellier, Cedex 5, France. nicola.marchi@igf.cnrs.fr. Conflict of interest. The Authors have no financial or conflict of interest to declare.

Publisher's Disclaimer: This is a PDF file of an unedited manuscript that has been accepted for publication. As a service to our customers we are providing this early version of the manuscript. The manuscript will undergo copyediting, typesetting, and review of the resulting proof before it is published in its final citable form. Please note that during the production process errors may be discovered which could affect the content, and all legal disclaimers that apply to the journal pertain. 
Results - In vivo, severe status epilepticus (SE) altered NG2DsRed vascular coverage. We found dishomogenous NG2DsRed perivascular ramifications after SE and compared to control. Concomitantly, PDGFR $\beta^{+}$cells re-distributed towards the cerebrovasculature after severe SE. Cerebrovascular NG2DsRed partially colocalized with PDGFR $\beta^{+}$while parenchymal PDGFR $\beta^{+}$ cells did not colocalize with IBA- $1^{+}$microglia. Using in vitro $\mathrm{OHC}$ we found decreased NG2 vascular staining and increased PDGFR $\beta^{+}$ramifications associated with RECA- $1^{+}$microvessels after seizure-like activity. Cellular PDGFR $\beta$ and $\mathrm{NG}^{+}$colocalization was observed in the parenchyma. Finally, analysis of human TLE brains revealed perivascular and parenchymal PDGFR $\beta^{+}$cells distribution resembling the murine in vivo and in vitro results. PDGFR $\beta^{+}$cells at the cerebrovasculature were more frequent in TLE brain tissues as compared to the autoptic control.

Conclusions-The rearrangement of PDGFR $\beta^{+}$and vascular NG2DsRed cells after SE suggest a possible involvement of pericytes in the cerebrovascular modifications observed in epilepsy. The functional role of vascular-parenchymal PDGFR $\beta^{+}$cell redistribution and the relevance of a pericyte response to SE remain to be fully elucidated.

\section{Keywords}

pericytes; PDGFR $\beta$; NG2DsRed; blood-brain barrier; status epilepticus

\section{Introduction}

An increasing body of evidence is in favor of an etiological role of blood-brain barrier (BBB) dysfunction in seizure disorders [1-3]. Damage of the BBB in epilepsy is accompanied by aberrant angiogenesis and the formation of leaky microvessels [4]. BBB damage allows for the extravasation of serum components into the brain parenchyma, altering the composition of the brain milieu [5]. Pathophysiological changes occurring in BBB endothelial cells and astrocytes have been identified as culprits of BBB dysfunction during seizures $[3,6]$.

In addition to astrocytes and endothelial cells, vascular mural cells, such as pericytes, significantly contribute to BBB physiology and integrity [7-9]. Interestingly, the pathophysiological role of pericytes in brain disorders has begun to attract attention [7]. Pericytes are located in the perivascular space of the BBB, connected by tight and gap junction proteins. Pericytes form a tight sleeve around the cerebral vessels and can be distinguished by their association with microcapillaries $[7,8,10]$. Under normal conditions, pericytes cover approximately $60-80 \%$ of the cerebrovascular tree, controlling BBB functions. Pericyte deficient mice have BBB leakage [11-13], demonstrating the requirement of pericytes for proper cerebrovascular integrity $[14,15]$.

A number of cellular markers and molecular tools have been used to identify pericytes. Recent evidence has indicated that transgenic mice expressing DsRed under control of the proteoglycan NG2 promoter (NG2DsRed) can be used to visualize cerebrovascular pericytes $[16,17]$. In this model, NG2 fluorescence is detected only in brain vascular mural cells but not in mature parenchymal oligodendrocytes (or polydendrocytes), as instead previously reported using other models $[18,19]$. NG2 ${ }^{+}$pericytes were demonstrated to play an 
important role in the developing cerebrovasculature [16]. Loss of $\mathrm{NG}^{+}$pericytes was shown to reduce pericyte-endothelial cross-talk and the expression of the tight junction protein ZO1 [20].

The platelet derived growth factor receptor beta (PDGFR $\beta$ ) has also been proposed as a marker of pericytes [7, 9, 21, 22]. PDGFR $\beta$ is involved in the molecular cross-talk between pericytes and endothelial cells [22]. PDGFR $\beta$, and its ligand PDGF-BB, play a pivotal role in pericyte recruitment during brain development [7]. Impaired PDGFR $\beta$ signaling leads to pericyte functional deficiency and severe neuro-vascular impairments [11, 12, 23]. However, controversy exists on PDGFR $\beta$ cellular specificity. It has been proposed that PDGFR $\beta$ is expressed by perivascular pericytes and play a key role in cell recruitment during vascular remodeling [7, 22]. In addition, increased vascular and parenchymal PDGFR $\beta^{+}$cells were found in the brain in response to acute stroke $[24,25]$ or in the heart after ventricular overload [26]. PDGFR $\beta$ expression has been proposed as a rescue response to a vascular pathological insult [26].

Despite the recent interest in the role of pericytes in brain pathologies, sparse data exist on their involvement in epilepsy [27]. We have herein investigated the effect of experimentally induced status epilepticus on vascular pericytes using NG2DsRed and PDGFR $\beta$ as markers. We have also investigated the effect of status epilepticus on PDGFR $\beta^{+}$cells in the parenchyma. We have used in vitro organotypic hippocampal cultures to substantiate the in vivo data and evaluated the pattern of PDGFR $\beta$ expression in a cohort of human brain resections obtained from temporal lobectomies performed to relieve seizures.

\section{Methods}

\section{Human tissue}

Brain specimens were obtained conforming to the principles outlined in the Declaration of Helsinki and in accordance with the French Ethical Committee and the University Hospital of Montpellier (Pathological Department, Centre des Collections Biologiques Hospitalières de Montpellier CCBH-M, Collection tumorothèque, FINESS 340780477, F-34285

Montpellier, France). All patients (or their families) were informed of additional studies performed on surgical tissue and provided consent. We used brain sections (formalin fixed, paraffin embedded) derived from 5 patients (age: $34 \pm 13$ years, see Table 1) affected by intractable temporal lobe seizures associated with hippocampal sclerosis and 1 autoptic brain derived from a patient affected by pulmonary cancer without neurological disorders.

\section{Animals}

All animal procedures were conducted in accordance with the European Communities Council Directive (86/609/EEC) and approved by the Ministere de la Recherche Française (protocol 00846.01). Animals were housed in a controlled environment $\left(21 \pm 1^{\circ} \mathrm{C}\right.$; humidity 60\%; lights on 08:00 AM - 8:00 PM; food and water available ad libitum). 


\section{Seizure induction and monitoring}

Status epilepticus (SE) was induced in C57BL/6J mice by intra-peritoneal injections of kainic acid (KA, $10 \mathrm{mg} / \mathrm{ml}$ in PBS; $25 \mathrm{mg} / \mathrm{kg}$, Sigma). The same dosage was used in NG2Dsred transgenic animals (http://jaxmice.jax.org/strain/008241.html). Time of SE onset was comparable in the two animal models (25-30 minutes). All animals were scored according to the Racine Scale. Animals were considered in SE when a generalized tonic-clonic event associated with a loss of balance was observed (Stage VI Racine). We considered animals having severe $\mathrm{SE}$ when $>5$ events of tonic -clonic generalized convulsions (each lasting 30-45 seconds over a period of 90 minutes) were observed. A subset of C57BL/6J mice ( $\mathrm{n}=$ 5) was implanted with cortical EEG electrodes to confirm the electrographic correlate (not shown) of behavioral SE.

\section{Animal usage}

A total of 18 NG2-DsRed (C57BL/6J background; male, age 8-12 weeks) transgenic mice were injected i.p. with KA $(25 \mathrm{mg} / \mathrm{kg})$. Mortality was of approximately $40-50 \%$. One animal reached Stage IV/V and was not included in the SE group. The remainder of the animals developed a severe SE. At this stage, mortality was $20 \%$ and SE was not alleviated using benzodiazepines. Five NG2-DsRed mice were injected with saline and used as control. We obtained $\mathrm{n}=7$ NG2-Dsred mice experiencing severe SE (Figures 1, 3 and Supplemental Figure 1). Animals were sacrificed 24 hours after SE onset.

We used 12 male C57BL/6J mice (8-12 weeks). Mice were injected with KA (25 mg/kg). Three animals did not develop severe SE (indicated as Stage IV/V in Figure 4C-C1). Mortality was approximately $30 \%$. We obtained a total of $\mathrm{n}=5$ mice experiencing SE. Animals were sacrificed 24 hours after SE. Five additional C57BL/6J mice were used as control and injected with sterile saline (Figures 2, 4 and Supplemental Figure 1). Animals (NG2DsRed or C57BL/6J) were injected intracardially with 250-300 $\mu$ l of FITC-Dextran or FITC-Albumin solutions (Sigma-Aldrich; $25 \mathrm{mg} / \mathrm{ml}$ in PBS) and brains removed after 1-2 minutes. Dextran and albumin have comparable molecular weight (50-60 KDa). Additional C57BL/6J KA mice $(\mathrm{n}=3)$ were injected intracardially with Evans Blue (2\% in PBS;

Supplemental Figure 2). Brains were fixed (immersion) in $4 \%$ formalin for at least 48 hours.

\section{Organotypic slices}

Methods were previously described [4]. We used 3 separate OHC cultures, each obtained using 7-8 pups. Briefly, hippocampi from 6 - 7 day old Sprague Dawley rats (Janvier) were dissected under aseptic conditions, and transverse sections $(400 \mu \mathrm{m})$ were obtained using a tissue chopper. Slices were placed on a $30 \mathrm{~mm}$ porous membrane (Millicell-CM, Millipore) and kept in $100 \mathrm{~mm}$ diameter Petri dishes filled with $5 \mathrm{ml}$ of culture medium composed of $25 \%$ heat inactivated horse serum, 25\% HBSS, 50\% MEM, $25 \mathrm{U} / \mathrm{ml}$ penicillin, $25 \mu \mathrm{g} / \mathrm{ml}$ streptomycin (Invitrogen). Cultures were maintained in a humidified incubator at $36^{\circ} \mathrm{C}$ and $5 \% \mathrm{CO} 2$. Two days later, media was changed and the temperature set to $33^{\circ} \mathrm{C}$. After 2 weeks, the membranes were transferred to 6 well plates and each well was filled with $1 \mathrm{ml}$ of medium. Slices were treated for 1 hour with $25 \mu \mathrm{M}$ kainic acid (KA, Sigma-Aldrich). Field potential activity (CA1) was performed to evaluate onset of electrographic activity (see also [4]). Slices were then placed in KA free media for 24-72 hours. 


\section{Immunohistochemistry}

Human-Brain sections ( $4 \mu \mathrm{m}$ ) were obtained from blocks of resected tissues. After quenching of endogenous peroxidase, antigen retrieval was performed by immersion in citrate buffer at $\mathrm{pH}=6$. Tissues were incubated with an anti-PDGFR $\beta$ primary antibody (ab32570 Abcam, 1:100). The biotinylated secondary antibody was raised against rabbit IgG and used at 1:500 dilution. The immunoperoxidase reaction was performed using the avidinbiotin method and diaminobenzidine. This method was performed with the Venting automatic immunoassaying system (Ventana Benchmark Ultra, AR). Sections were counterstained with haematoxylin.

Animals-Brain sections $(30 \mu \mathrm{m})$ were placed on glass slides and rinsed twice with PBS. Blocking solution consisted of 3\% BSA and Triton $0.25 \%$ in PBS (1 hour at room temperature). Slices were then incubated with primary antibodies (1:100 rabbit monoclonal anti-PDGFR $\beta$, Abcam, ab32570; or 1: 1000 chicken polyclonal anti-GFAP, Abcam, ab4674; or 1:2000 goat anti-IBA1 Abcam, ab5076) in blocking solution (2 hours at room temperature). Secondary antibodies were chosen according to the primary host: donkey antirabbit IgG Alexa Fluor-568 1:1000 (Life Technologies; A10042); donkey anti-chicken IgG (Jackson Immunoresearch; AMCA); donkey anti-goat Alexa Fluor 555 (Invitrogen 1:2000) or donkey anti-rabbit AMCA (1:100). Incubation was performed at room temperature (2 hours). After washes with PBS, DAPI (Sigma; 1:500) was added and slices mounted with Mowiol. Images $(10 \times, 20 \times$ and $40 \times$ ) were acquired by fluorescent microscopy (Zeiss, AxioImager Z1) and processed using Photoshop CS6 and ImageJ.

Organotypic hippocampal culture. Slices were fixed using 4\% PFA (30 minutes). OHC were washed with PBS 3 times (10 minutes). Slices were then placed in blocking solution (20\% horse serum and Triton $0.5 \%$ in PBS) for 2 hours at room temperature. Slices were incubated for 48 hours with a rabbit anti-PDGFR $\beta$ primary antibody (Abcam 32570; 1:100) or mouse anti-RECA-1 (AbD Serotec MCA970R; 1:1000) or mouse anti-NG2 (Abcam, ab50009; $1: 200)$ in Triton $0.25 \%$ and PBS. Slices were then washed 3 times in PBS and secondary antibody: donkey anti-rabbit Alexa fluor 568 Invitrogen A10042; or goat antimouse Alexa fluor 488 Molecular Probes A11029; or donkey anti-mouse Alexa fluor 568 Life Technologies A10037. All secondary antibodies were added at 1: 1000 (2 hours at room temperature). Slices were then washed and mounted.

\section{Fluorescence quantification and 3D reconstruction}

NG2DsRed and PDGFR $\beta$ labeling (10x) were imaged using a fluorescent microscope (Zeiss, AxioImager Z1). Within sessions (NG2DsRed or PDGFR $\beta$ acquisition), identical exposure parameters (luminosity, gain and contrast) were maintained. We have selected areas from cortices (parietal and temporal) and dorsal/ventral hippocampal regions. DAPI staining was used to ensure anatomical correspondence between groups (control and postSE). Post-acquisition image analysis was performed using ImageJ. Briefly, brightness and contrast were modified on all pictures $(+150 \%$ and $+100 \%$ respectively) to facilitate automated detection of fluorescence. Specific signal was isolated by removing the background noise and keeping tolerance at 25 throughout all the images. Area (pixel) of NG2DsRed and PDGFR $\beta$ signals was then automatically measured. The same procedure 
was used to quantify intravascular FITC-Albumin signals (cerebrovascular tree). Images shown in Figures 1 and 2 are not representative of FITC leakages due to image processing (see Supplemental Figure 2). When a complete lack (e.g., entire slice, independent of the region) of FITC cerebral perfusion was observed, the slice was not included in the quantification. Data in Figures 1 and 2 are expressed as NG2DsRed or PDGFR $\beta$ pixels normalized by FITC-Albumin pixels $\times 100$. Quantification of percentage NG2DsRed/FITC (Figure 1) and PDGFR $\beta /$ FITC (Figure 2) coverage was calculated using $5 \mathrm{KA}+3$ controls (NG2-DsRed) and $5 \mathrm{KA}+5$ controls (C57BL/6J) respectively. FITC perfusion data (Supplemental Figure 2) refers to all mice (NG2-DSRed and C57BL/6J) intra-cardially perfused with FITC tracers.

Quantification of OHC signal-We used control and KA-treated hippocampal slices obtained from 3 separate cultures. Images (PDGFR $\beta$, NG2 and RECA-1; $5 \times$ ) were acquired using a fluorescence microscope (Zeiss, AxioImager Z1). All images were acquired using identical exposure parameters. Images were then processed using ImageJ. Briefly, images were converted into 8 bit black and white mode to allow a clear visualization of the signals. We use the segmented line tool to manually draw each vessel (PDGFR $\beta, N G 2$ and RECA-1). ROI manager was used to collect data. Length is expressed as pixels and then converted in $\mu \mathrm{m}(0.768$ pixel $=1 \mu \mathrm{m})$. Data were extracted in an excel file. Box plots in Figure 5 are expressed as relative changes to mean control value (100\%).

Confocal microscope analysis was performed using a sequential laser scanning confocal microscope (Zeiss LSM780). Double- or triple-labeled images from each region of interest were obtained using: green $480-534 \mathrm{~nm}$; red $575-620 \mathrm{~nm}$, blue $415-470 \mathrm{~nm}$. The objective and the pinhole setting remained unchanged during the acquisition of all images (40x). Post-acquisition processing and 3D reconstruction were performed using IMARIS 7.2. The resulting objects can be rendered with specific light properties and rotated in any direction, allowing for the visualization of cell-to-cell positioning (see Supplemental Movies).

\section{Statistical analysis}

We used Origin 8.0 (Origin Lab, Northampton, MA, USA) for all statistical analyses. Shapiro-Wilk test was used to evaluate the normal distribution of the data. Data are indicated as mean $\pm \mathrm{sem}$. One-way ANOVA (Bonferroni correction) was used on paired populations (e.g., CTR vs. post-SE). p $<0.05$ was considered statistically significant. Statistical Power was performed and results are provided in each Figure legend. Data are expressed using a Statistical Box Chart showing mean \pm SD, $5 \%$ and $95 \%$ percentile and individual data distribution.

\section{Results}

\section{Changes in capillary NG2DsRed pericytes after severe SE}

We used NG2-Dsred transgenic mice to study the pattern of pericyte vascular coverage following severe status epilepticus induced by kainic acid. Micro-angiography with FITC markers was used to visualize the cerebrovascular tree. In control animals, NG2DsRed cells 
covered the perivascular space of penetrating microvessels and parenchymal microcapillaries (Figure 1A and C; Supplemental Movie 1). We found $60-80 \%$ of the vasculature to be covered by NG2DsRed pericytes. Pericyte soma and ramifications lining the vascular wall were distinguishable across the brain regions analyzed (Supplemental Figure 1 and Supplemental Movie 1). Following severe SE (see methods), disarrangement of NG2DsRed pericytes was observed. Morphological changes spanned from a dishomogenous NG2DsRed-vascular interface to a loss of pericyte perivascular ramifications in specific aspects of the cerebrovasculature (Figure 1, Supplemental Figure 1 and Supplemental Movie 2). Quantification of NG2DsRed/FITC fluorescence indicated a reduction in NG2DsRed vascular coverage after severe SE (Figure 1B).

\section{Parenchymal and vascular PDGFR $\beta^{+}$cell redistribution after severe SE}

Under physiological conditions, C57BL/6J mice displayed PDGFR $\beta^{+}$cells in the brain parenchyma and associated with microvessels (Figure 2A-A2 and Figure 3A). Following severe SE we found increased PDGFR $\beta^{+}$vascular coverage (quantified in Figure $2 \mathrm{~B}$ as area PDGFR $\beta$ / FITC). PDGFR $\beta^{+}$cells redistribution was observed in the hippocampus and in the cortices in mice experiencing severe SE. Three-dimensional confocal reconstruction depicts examples of PDGFR $\beta^{+}$cells populating the vessels of the dentate gyrus (Supplemental Figure 1). Supplemental Movies 3 and 4 show 3D spatial arrangement of typical vessels. Note that mice reaching stage IV/V of the Racine's Scale did not display significant changes in PDGFR $\beta$ distribution (Figure 4C-C1).

\section{Pattern of PDGFR $\beta$ and NG2DsRed colocalization in vivo}

In control mice NG2DsRed pericytes partially colocalized with vascular PDGFR $\beta$ (Figure 3A). Parenchymal PDGFR $\beta^{+}$cells were also detected. Magnification in Figure 3B shows an example of NG2DsRed / PDGFR $\beta$ vascular arrangement. Note the presence of defined NG2DsRed soma and cellular processes colocalizing with PDGFRb. Following severe SE we found an increase in vascular PDGFR $\beta$ signal (Figure 3A1-A2 and B) and disorganized NGDsRed ramifications (see also Figure 1). These data suggest the presence of constitutive NG2DsRed / PDGFR $\beta^{+}$vascular pericytes and the possible recruitment of PDGFR $\beta^{+}$cells occurring after severe SE. In addition, parenchymal PDGFR $\beta+$ cells did not colocalize with the microglial marker IBA-1 (Figure 4A) while NG2DsRed cells did not colocalize with GFAP (Figure 4B).

\section{Redistribution of PDGFR $\beta^{+}$and $\mathrm{NG}^{+}$cells in $\mathrm{OHC}$ after seizure-like activity}

We evaluated whether changes observed in vivo could be reproduced in vitro using organotypic hippocampal cultures ( $\mathrm{OHC}$, Figure 5). Exposure of $\mathrm{OHC}$ to KA triggered seizure-like activity (as previously reported in [4]) consisting of a train of 8-10 Hz spikes, each lasting 10 to 20 seconds over a period of 15-20 minutes. No activity was recorded after media washout, as well as in non-treated slices. Following seizure-like activity we found a redistribution of PDGFR $\beta$ cells consistent with the results shown in Figures 2 and 3. In particular, Figure 5A shows morphological changes with PDGFR $\beta^{+}$cells lining the vasculature after in vitro seizure-like activity (KA) compared to control. PDGFR $\beta^{+}$ immunoreactivity lining the cerebrovasculature was increased after KA treatment while 
vascular RECA-1 was comparable in control and KA-treated slices (Figure 5B1-B2). Figure 5B shows magnifications of PDGFR $\beta^{+}$cells distributed at the RECA- 1 cerebrovasculature. Note the proximity of PDGFR $\beta$ and RECA-1 signal at the cerebrovasculature after in vitro seizure-like activity. Following in vitro seizure-like events, reduction and disorganization of NG2 vascular signal was also observed (Figure 5B3 and C-C1). This result is in accordance with the in vivo data obtained using NG2DsRed mice (see Figure 1). Examples of NG2 immunostaining show vascular and parenchymal NG2+ cells (Figure $5 \mathrm{C}-\mathrm{C} 1$ ). The latter data is in accordance with previous reports where staining of parenchymal NG2+ cells was observed $[18,19]$. We found increased co-localization between NG2 and PDGFR $\beta$ in the parenchyma after SE (Figure 5C1). The exact nature of parenchymal NG2/PDGFR $\beta$ positive cells remains to be fully elucidated.

\section{Pattern of PDGFR $\beta$ expression in human TLE}

No data are available describing the brain expression of PDGFR $\beta$ in patients affected by refractory epilepsy (TLE, see Table 1). We found PDGFR $\beta^{+}$cells associated with vessels and in the parenchyma of control tissue (Figure 6A-A1). Analysis of the TLE brain samples indicated heterogeneous patterns of PDGFR $\beta$ immunoreactivity. In particular, PDGFR $\beta^{+}$ cells were increased at the cerebrovasculature in 3 out of the 5 TLE specimens analyzed and compared to the available control. In some cases, ramified parenchymal PDGFR $\beta^{+}$cells projected to the vessels (Figure 6B). Figure 6C also shows an example of a brain TLE sample that had a moderate PDGFR $\beta$ increase as compared to control. Panel D shows an example of tissue used as a positive control for PDGFR $\beta$ expression (human kidney). The results obtained using TLE brain specimens and an experimental model of acute seizures hint to a redistribution of PDGFR $\beta^{+}$pericytes during seizures.

\section{Discussion}

Our results indicate that: i) constitutive cerebrovascular NG2DsRed pericyte coverage is impaired in response to $\mathrm{SE}$ in vivo or seizure-like events in vitro; ii) concomitantly, a redistribution of parenchymal and vascular PDGFR $\beta^{+}$cells occurs in vitro and in vivo; iii) vascular and parenchymal PDGFR $\beta^{+}$cells partially colocalize with NG2DsRed and NG2 but not with IBA-1; iv) PDGFR $\beta^{+}$cells are distributed around the cerebrovasculature and are present in the brain parenchyma of human TLE specimens. PDGFR $\beta$ immunoreactivity was robust in TLE samples as compared to autoptic control brain.

Pericytes can be identified using a combination of markers and their anatomical position with microcapillaries. The perivascular localization and the morphological changes of NG2DsRed and PDGFR $\beta^{+}$cells found in our study suggest an involvement of pericytes in epilepsy. Concomitantly we detected PDGFR $\beta^{+}$and $\mathrm{NG}^{+}$cells in the brain parenchyma, suggesting other possible origins of these cells $[18,24]$. Thus, controversy exists whether PDGFR $\beta^{+}$is exclusively expressed by perivascular pericytes [28] or by parenchymal cells [25] and proliferating brain-resident stromal cells [24]. It remains uncertain whether PDGFR $\beta$ indicates multiple cell types (pericyte-like) at different locations (vascular and parenchymal). Similar considerations may apply to $\mathrm{NG}^{+}$cells (vascular and polydendrocytes) in the brain [18] 


\section{Pericytes, BBB dysfunction and seizures}

Pericytes have increasingly gained attention owing to their role in cerebrovascular development, brain pathophysiology and angiogenesis $[7,15,16]$. While perivascular pericytes have been studied in neurodegenerative disorders and stroke [12, 24, 29], scarce information is available on their response to seizures. Our initial findings introduce pericytes as possible players in the cerebrovascular dysfunction characteristic of seizure disorders. In accordance with other reports [7, 15, 16, 22], we have used PDGFR $\beta$ and NG2DsRed mice to identify pericytes surrounding cerebral vessels. The rearrangement of perivascular pericytes observed after SE supports the role of cerebrovascular dysfunction in epilepsy [3, 13]. Blood-brain barrier damage was demonstrated to trigger [30] or sustain seizure activity [1] and pericyte loss could contribute to the multi-cellular failure occurring at the epileptic BBB.

In addition, it was recently proposed that an increased PDGFR $\beta$ cellular response could counterbalance the initial loss of capillary pericytes following cerebral ischemia [24]. We now propose that a similar mechanism could take place after SE. Several questions remain, however, unanswered. For instance, it is unclear whether pericytes transiently upregulate PDGFR $\beta$ during vascular redistribution. In addition it is unclear whether pericyte subpopulations with distinct functions and topography exist, reacting differently to pathological stimuli. The latter hypothesis needs further investigation as PDGFR $\beta$ (and NG2) immunoreactivity is observed at the vascular and parenchymal levels [7, 18, 22, 24, 28].

Limited information is available describing pericyte changes in brain samples obtained from patients affected by refractory epilepsy. One study has shown pericyte morphological changes and the thickening of the basal cerebrovascular membrane in human TLE [27]. Our results are novel as they represent the first attempt to determine PDGFR $\beta$ pericyte expression in capillaries and penetrating vessels in TLE brains (Figure 6). Others have observed a trend increase of PDGFR $\beta^{+}$cells in the abnormal white matter (human focal cortical dysplasia), as compared to correspondent control regions [31]. Interestingly, administration of the PDGFR $\beta$ ligand PDGF-BB suppresses convulsions in a mouse model [32]. The effect was ascribed to a direct functional modification of hippocampal neurons. Whether changes in the pericyte-endothelial cells interface could be involved remains to be determined.

\section{Vascular / parenchymal NGDsRed, NG2 and PDGFR $\beta$ redistribution after SE}

In our study we have used a combination of transgenic mice and cellular markers to investigate the response of vascular pericytes to severe SE. NG2DsRed cells at the vasculature identify pericytes (Figures 1, 3 and 4) while immunohistochemistry against NG2 showed both vascular and parenchymal cells (Figure 5). In our hands, NGDsRed mice constitute an adequate experimental tool to investigate vascular pericyte fragmentation in response to pathological conditions. Moreover, our results are in accordance with previous evidence suggesting multiple sites of PDGFR $\beta$ expression [7, 24, 25, 28]. A preliminary evaluation of the redistribution of PDGFR $\beta^{+}$cells occurring in vivo 3 days after SE indicated an increased in the parenchymal and vascular cell number (not shown). The latter data 
support a time dependent PDGFR $\beta^{+}$cell response and redistribution after SE. We also found that a number of NG2DsRed or NG2 pericyte colocalized with PDGFR $\beta$ at the vasculature (Figures 3 and 5). Available evidence has shown co-localization of NG2 and PDGFR $\beta$ in pericytes associated with the cerebrovasculature in the developing human brain [16].

The parenchymal and vascular PDGFR $\beta$ rearrangement consequent to SE is in accordance with available data obtained using a model of ischemia stroke [24]. Moreover, the pattern of PDGFR $\beta$ immunostaining herein reported was qualitatively similar between experimental approaches (in vivo Figures 2-3; in vitro Figure 5; human TLE Figure 6). A comprehensive study is required to elucidate the pathophysiological and clinical determinants of PDGFR $\beta$ expression in the human epilepsy. It is important to underscore that, under physiological conditions, pericytes directly communicate with cerebrovascular endothelial cells by cell-tocell junction proteins, including cadherins and connexins [8]. Whether changes in pericyteendothelial adherens junctions occur after SE remains to be evaluated.

Our data (Supplemental Figure 2) suggests localized changes in cerebrovascular permeability and FITC-albumin intravascular distribution post-SE [1, 2]. We found a decrease of signal in the sub-cortical regions of frontoparietal and temporal poles after SE [33]. Hippocampal regions did not display significant differences except for the dentate gyrus were a trend was observed. Remarkably, recent evidence has highlighted the role of pericytes in regulating brain hemodynamics and vascular tone in response to changes in neural activity $[17,34]$. Whether changes in perivascular pericytes contribute to the perfusion changes occurring during seizures remains to be elucidated. The pattern of NG2/ PDGFR $\beta$ vascular distribution and coverage in relation to cerebrovascular leakage also needs further investigation. The possibility exists that pericyte coverage could impact the regulation of the cerebrovascular tone during SE or alter the neuro-vascular coupling during inter-ictal to ictal transitions. The latter hypothesis needs experimental support.

\section{Conclusion}

In summary, our results are suggestive of a re-arrangement of perivascular pericytes occurring after severe SE. The use of specific pericyte-specific knock-out animals and extended monitoring after seizures will allow a more clear understanding of the observed vascular-parenchymal multicellular redistribution and the role of the pericyte-endothelial unit during seizures.

\section{Supplementary Material}

Refer to Web version on PubMed Central for supplementary material.

\section{Acknowledgments}

Supported by ANR AngEPI and NIH R01 NS078307-01.

\section{References}

1. van Vliet EA, et al. Longitudinal assessment of blood-brain barrier leakage during epileptogenesis in rats. A quantitative MRI study. Neurobiol Dis. 2014; 63:74-84. [PubMed: 24321435] 
2. Marchi N, et al. Blood-brain barrier dysfunction and epilepsy:pathophysiologic role and therapeutic approaches. Epilepsia. 2012; 53:1877-1886. [PubMed: 22905812]

3. Friedman A. Blood-brain barrier dysfunction, status epilepticus, seizures, and epilepsy: a puzzle of a chicken and egg? Epilepsia. 2011; 52(Suppl 8):19-20. [PubMed: 21967353]

4. Morin-Brureau M, et al. Epileptiform activity induces vascular remodeling and zonula occludens 1 downregulation in organotypic hippocampal cultures: role of VEGF signaling pathways. The Journal of neuroscience : the official journal of the Society for Neuroscience. 2011; 31:1067710688. [PubMed: 21775611]

5. Seiffert E, et al. Lasting blood-brain barrier disruption induces epileptic focus in the rat somatosensory cortex. The Journal of neuroscience : the official journal of the Society for Neuroscience. 2004; 24:7829-7836. [PubMed: 15356194]

6. Ivens $\mathrm{S}$, et al. TGF-beta receptor-mediated albumin uptake into astrocytes is involved in neocortical epileptogenesis. Brain. 2007; 130:535-547. [PubMed: 17121744]

7. Winkler EA, et al. Central nervous system pericytes in health and disease. Nat Neurosci. 2011; 14:1398-1405. [PubMed: 22030551]

8. Armulik A, et al. Pericytes: developmental, physiological, and pathological perspectives, problems, and promises. Dev Cell. 2011; 21:193-215. [PubMed: 21839917]

9. Armulik A, et al. Pericytes regulate the blood-brain barrier. Nature. 2010; 468:557-561. [PubMed: 20944627]

10. Dore-Duffy P, Cleary K. Morphology and properties of pericytes. Methods Mol Biol. 2011; 686:49-68. [PubMed: 21082366]

11. Sengillo JD, et al. Deficiency in mural vascular cells coincides with blood-brain barrier disruption in Alzheimer's disease. Brain Pathol. 2013; 23:303-310. [PubMed: 23126372]

12. Sagare AP, et al. Pericyte loss influences Alzheimer-like neurodegeneration in mice. Nat Commun. 2013; 4:2932. [PubMed: 24336108]

13. Marchi N, Lerner-Natoli M. Cerebrovascular remodeling and epilepsy. Neuroscientist. 2013; 19:304-312. [PubMed: 23072899]

14. Vates GE, et al. Pericyte constriction after stroke: the jury is still out. Nat Med. 2010; 16:959. author reply 960. [PubMed: 20823870]

15. Daneman R, et al. Pericytes are required for blood-brain barrier integrity during embryogenesis. Nature. 2010; 468:562-566. [PubMed: 20944625]

16. Virgintino D, et al. An intimate interplay between precocious, migrating pericytes and endothelial cells governs human fetal brain angiogenesis. Angiogenesis. 2007; 10:35-45. [PubMed: 17225955]

17. Hall CN, et al. Capillary pericytes regulate cerebral blood flow in health and disease. Nature. 2014; 508:55-60. [PubMed: 24670647]

18. Braganza O, et al. Albumin is taken up by hippocampal NG2 cells and astrocytes and decreases gap junction coupling. Epilepsia. 2012; 53:1898-1906. [PubMed: 22967085]

19. Wennstrom M, et al. Electroconvulsive seizures induce proliferation of NG2-expressing glial cells in adult rat hippocampus. Biol Psychiatry. 2003; 54:1015-1024. [PubMed: 14625143]

20. You WK, et al. NG2 proteoglycan promotes tumor vascularization via integrin-dependent effects on pericyte function. Angiogenesis. 2014; 17:61-76. [PubMed: 23925489]

21. Dore-Duffy P. Pericytes: pluripotent cells of the blood brain barrier. Curr Pharm Des. 2008; 14:1581-1593. [PubMed: 18673199]

22. Armulik A, et al. Endothelial/pericyte interactions. Circ Res. 2005; 97:512-523. [PubMed: 16166562]

23. Bell RD, et al. Pericytes control key neurovascular functions and neuronal phenotype in the adult brain and during brain aging. Neuron. 2010; 68:409-427. [PubMed: 21040844]

24. Fernandez-Klett F, et al. Early loss of pericytes and perivascular stromal cell-induced scar formation after stroke. J Cereb Blood Flow Metab. 2013; 33:428-439. [PubMed: 23250106]

25. Shen J, et al. PDGFR-beta as a positive regulator of tissue repair in a mouse model of focal cerebral ischemia. J Cereb Blood Flow Metab. 2012; 32:353-367. [PubMed: 21952111] 
26. Chintalgattu V, et al. Cardiomyocyte PDGFR-beta signaling is an essential component of the mouse cardiac response to load-induced stress. J Clin Invest. 2010; 120:472-484. [PubMed: 20071776]

27. Liwnicz BH, et al. Pericyte degeneration and thickening of basement membranes of cerebral microvessels in complex partial seizures: electron microscopic study of surgically removed tissue. Neurosurgery. 1990; 26:409-420. [PubMed: 2320209]

28. Winkler EA, et al. Pericyte-specific expression of PDGF beta receptor in mouse models with normal and deficient PDGF beta receptor signaling. Mol Neurodegener. 2010; 5:32. [PubMed: 20738866]

29. Winkler EA, et al. Blood-spinal cord barrier pericyte reductions contribute to increased capillary permeability. J Cereb Blood Flow Metab. 2012; 32:1841-1852. [PubMed: 22850407]

30. Marchi N, et al. Seizure-promoting effect of blood-brain barrier disruption. Epilepsia. 2007; 48:732-742. [PubMed: 17319915]

31. Shepherd C, et al. A quantitative study of white matter hypomyelination and oligodendroglial maturation in focal cortical dysplasia type II. Epilepsia. 2013; 54:898-908. [PubMed: 23551043]

32. Masuda Y, et al. Platelet-derived growth factor B-chain homodimer suppressing a convulsion of epilepsy model mouse El. Biochem Biophys Res Commun. 1996; 223:60-63. [PubMed: 8660379]

33. Fabene PF, et al. Pilocarpine-induced status epilepticus in rats involves ischemic and excitotoxic mechanisms. PLoS One. 2007; 2:e1105. [PubMed: 17971868]

34. Peppiatt CM, et al. Bidirectional control of CNS capillary diameter by pericytes. Nature. 2006; 443:700-704. [PubMed: 17036005] 


\section{Highlights}

- $\quad$ PDGFR $\beta^{+}$and vascular NG2DsRed cells redistribute after status epilepticus

- $\quad$ PDGFR $\beta$ colocalizes with NG2

- PDGFR $\beta^{+}$cells populate the parenchyma and the cerebrovasculature in human TLE

- Pericytes could be involved in the cerebrovascular dysfunction during seizures 

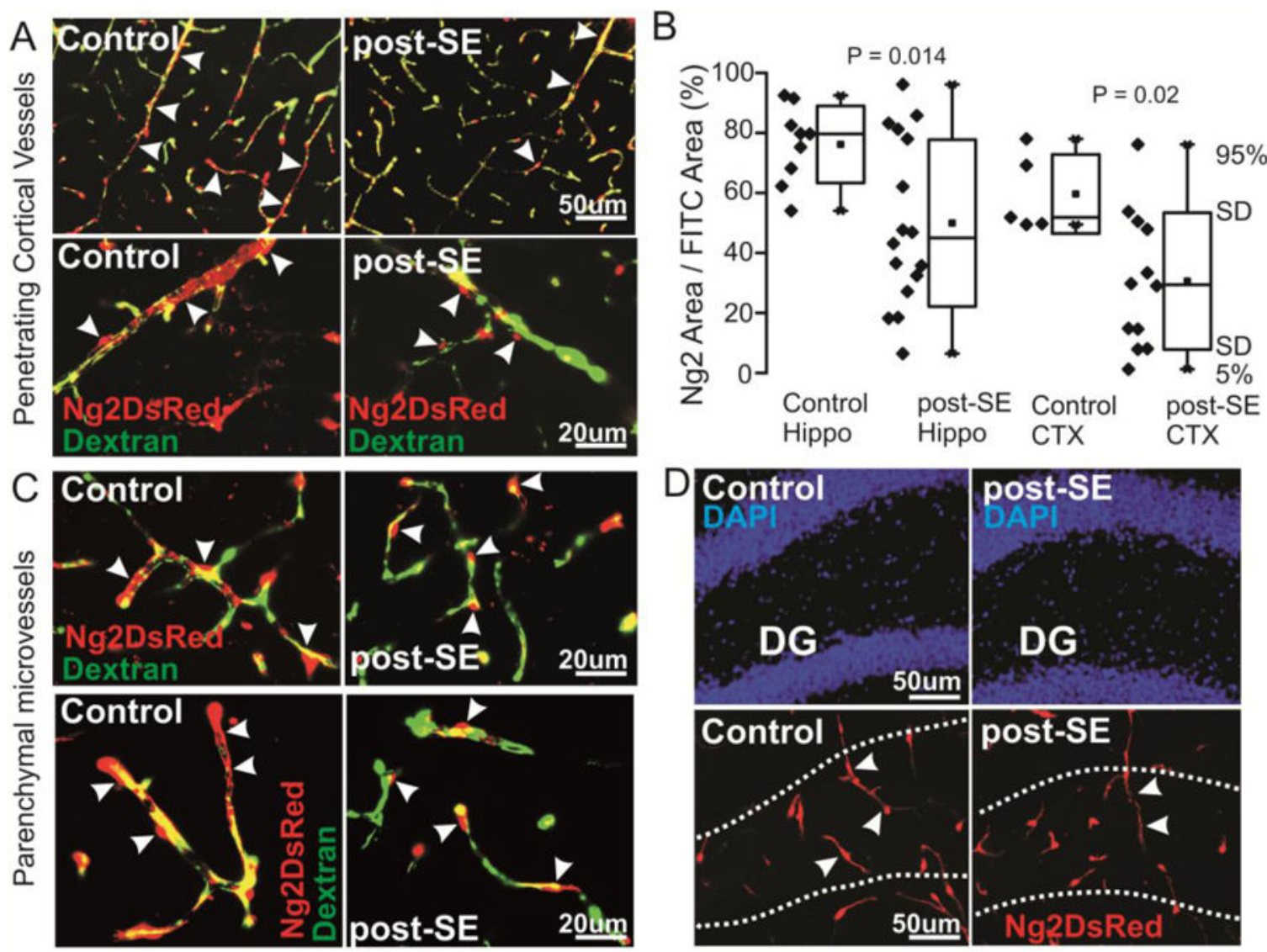

Figure 1. Pattern of NG2DsRed cerebrovascular coverage after severe $\mathrm{SE}$ in vivo

In control NG2DSRed transgenic mice, approximately $60-80 \%$ of the penetrating cortical microvessels (Panel A) and microcapillaries (Panel C) surface is covered by pericytes (Panel B). FITC-dextran was used to visualize the cerebrovascular tree. Following severe SE (post$\mathrm{SE}$ ), retraction of NG2DsRed pericyte ramification was observed. Arrowheads indicate pericyte soma. Changes were quantified (Panel B; see also methods for details) both in the hippocampus and cortices. An example of pericytes modification occurring in the dentate gyrus (DG) (arrowhead) is provided in Panel D. Results refer to $n=3$ control and $n=5 \mathrm{SE}$ NG2DsRed mice. Data for CA1, CA3 and DG (hippo) and parietal and temporal cortices $(\mathrm{CTX})$ are pooled together. $\mathrm{n}=9$ to 16 slices per condition. Power analysis: hippo $=72 \%$; CTX $=69 \%$. 


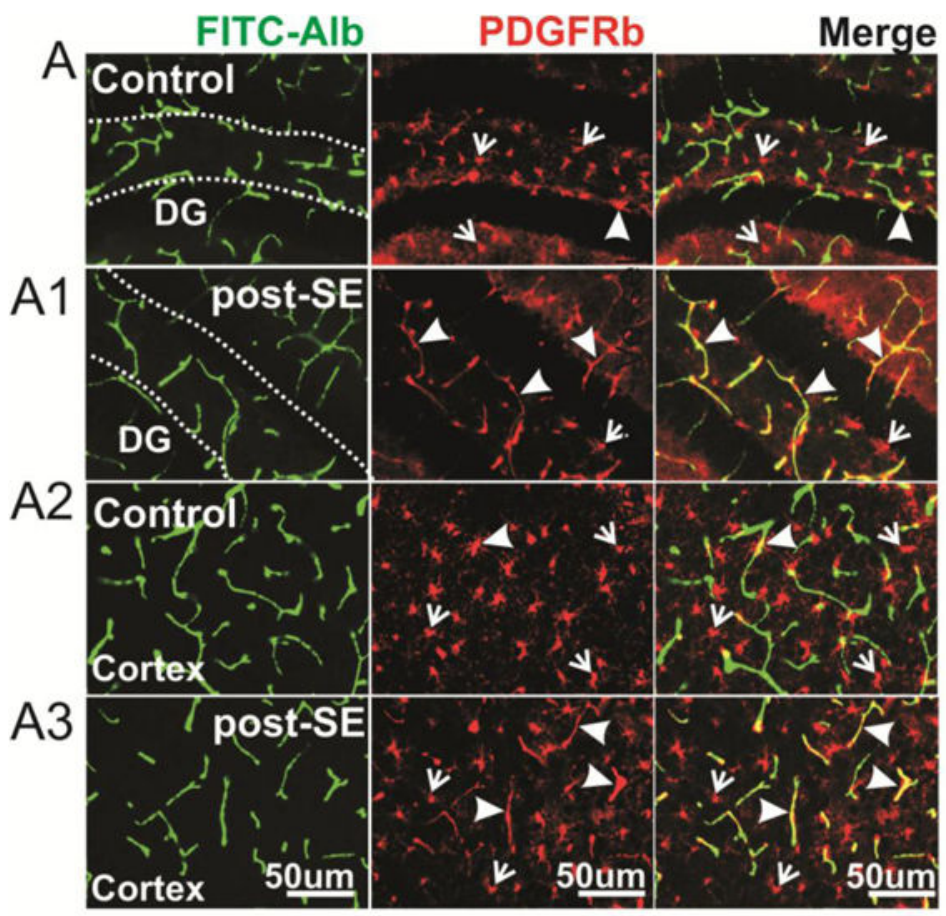

B

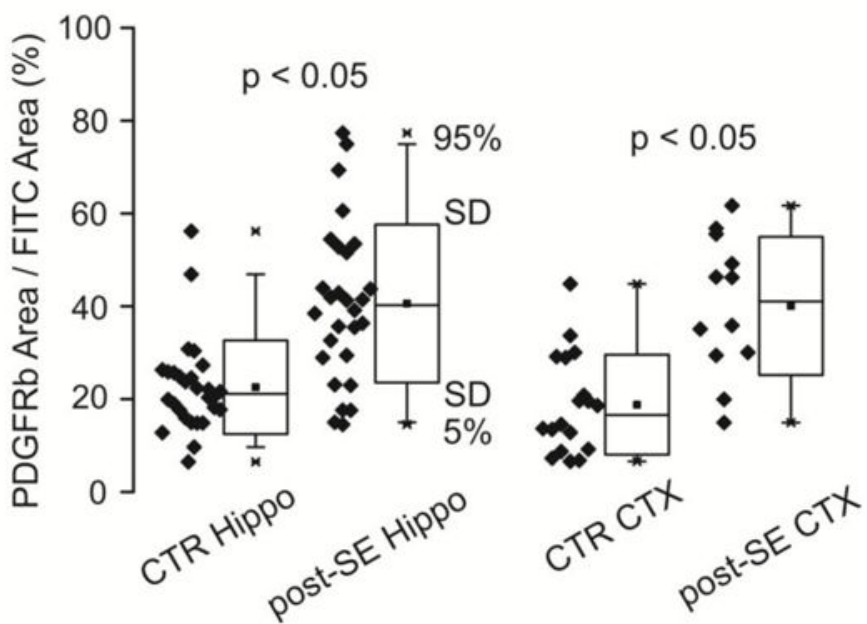

Figure 2. Vascular PDGFR $\beta^{+}$immunoreactivity following severe SE

A) PDGFR $\beta$ cells populate the cortex and hippocampus (DG is provided) of control C57BL/6J brain (arrows). Arrows indicate the PDGFR $\beta^{+}$cells that are distant from the vessels (visualized by FITC-albumin). Post-SE, PDGFR $\beta$ co-localize with the cerebrovasculature (arrow heads). See also Figure 3. B) Quantification of PDGFR $\beta^{+}$area overlapping with the cerebrovasculature (see also methods). Note the shift in PDGFR $\beta^{+}$ distribution/area between control and following SE. Data refer to: $n=5$ CTR and $n=5$ SE; 8-10 slices per condition. Data for CA1, CA3 and DG (hippo) are pooled together. Data for parietal and temporal cortex (CTX) are pooled together. Power: hippo and CTX $=90 \%$. 


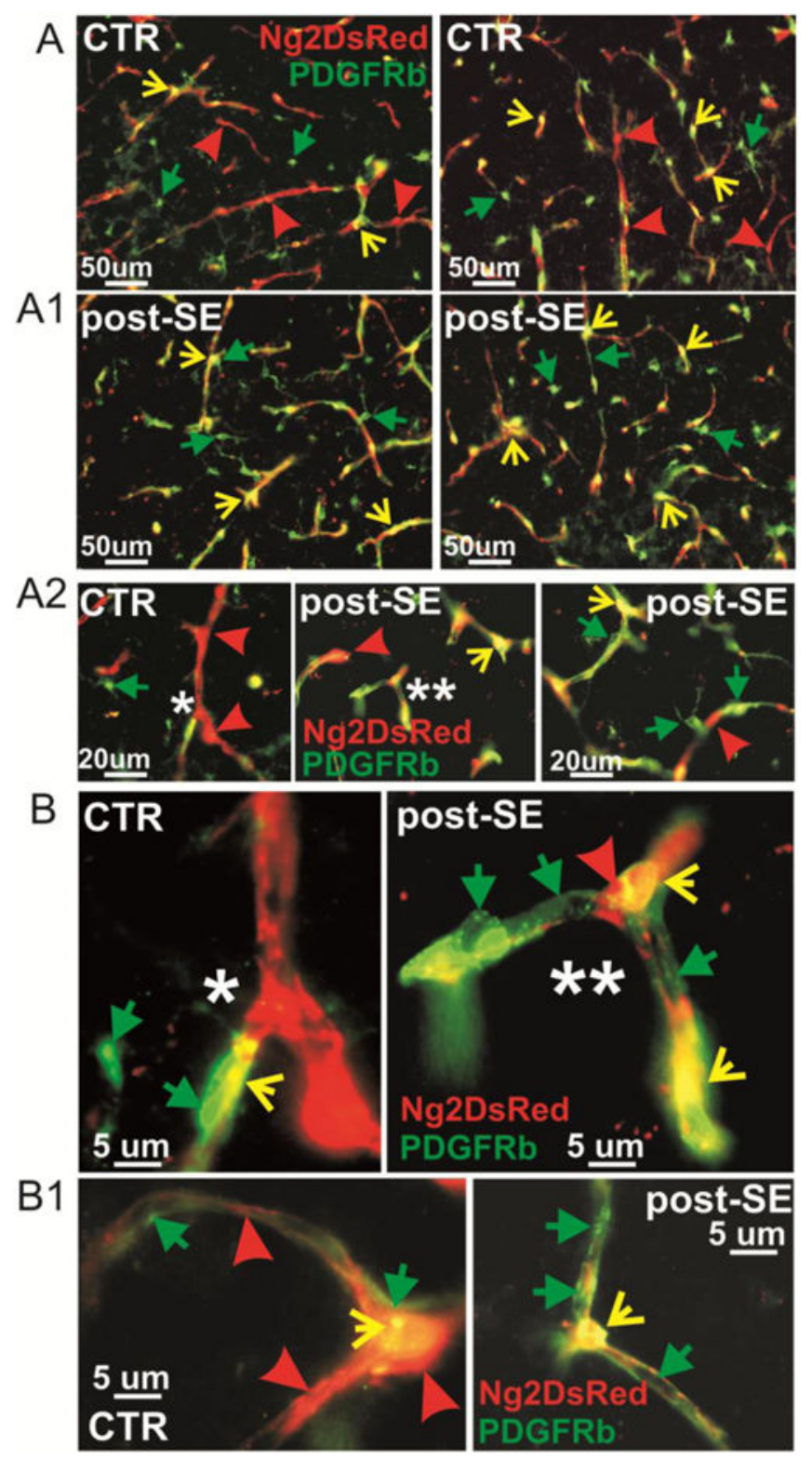

Figure 3. Pattern of NG2DsRed and PDGFR $\beta$ colocalization at the cerebrovasculature A) Examples of control animals. Note that the majority of the cerebral microcapillaries are covered by NG2DsRed pericytes (red arrows). PDGFR $\beta$ cells colocalize at the vasculature (yellow arrow) and are represented in the parenchyma (green arrow). A1) Post-SE increased PDGFR $\beta$ immunoreactivity and NG2DsRed/PDGFR $\beta$ colocalization is observed at the vasculature (green and yellow arrows). Note the presence of parenchymal PDGFR $\beta$ ramifications (green arrow). A2) Magnification of control and post-SE vessels. B-B1) Details of vascular distribution of NG2DsRed and PDGFR $\beta$ cells. 


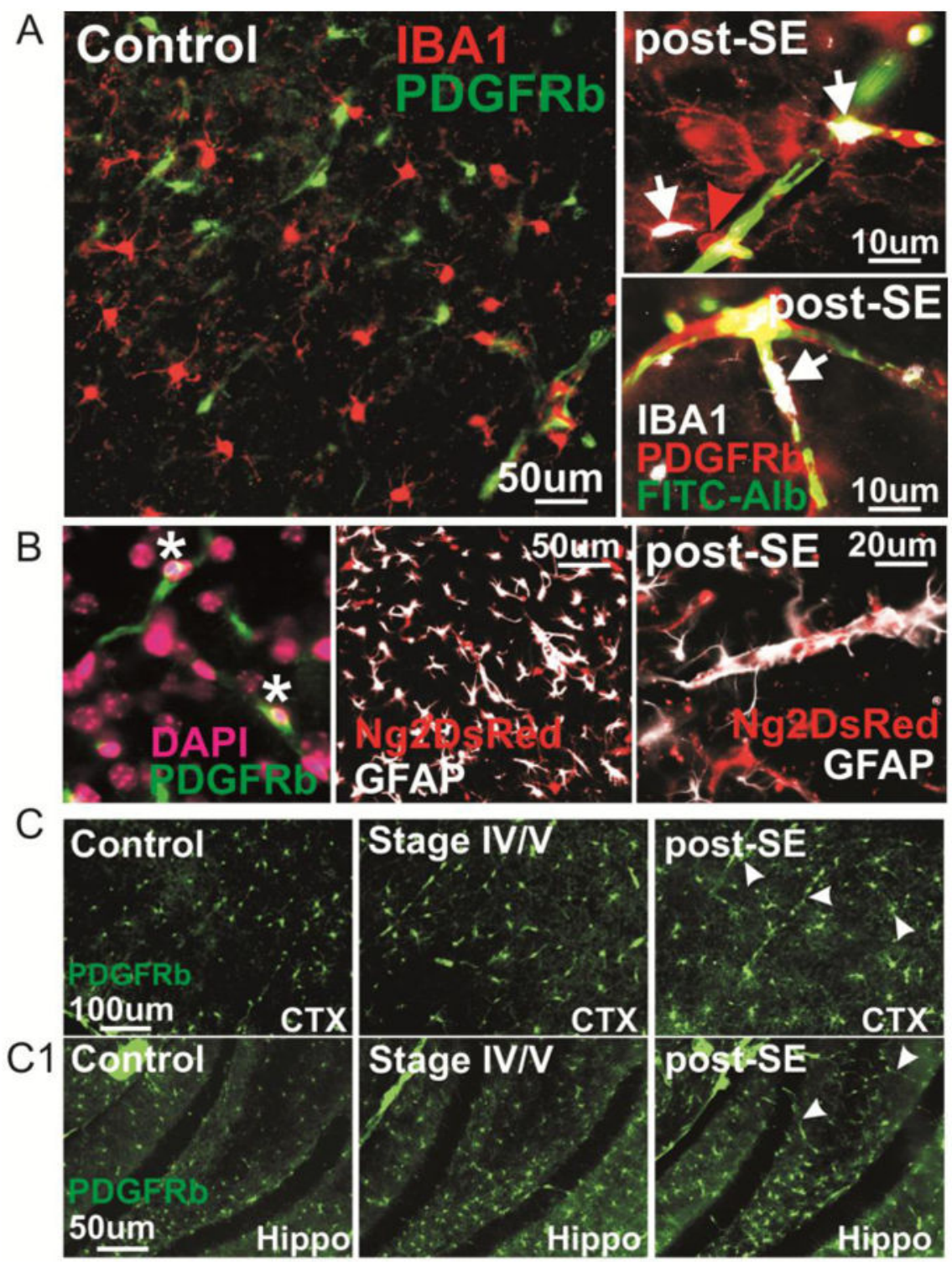

Figure 4. A) Lack of PDGFR $\beta$ colocalization with IBA-1

Vascular and parenchymal PDGFR $\beta+$ cells did not colocalize with microglia in control and post-SE; B) Example of PDGFR $\beta^{+}$soma (DAPI) at the cerebrovasculature. Moreover, NG2DsRed did not colocalize with GFAP. C-C1) Animal experiencing Stage IV/V behavioral seizures did not display changes in PDGFR $\beta^{+}$immunoreactivity and distribution compared to SE mice. 

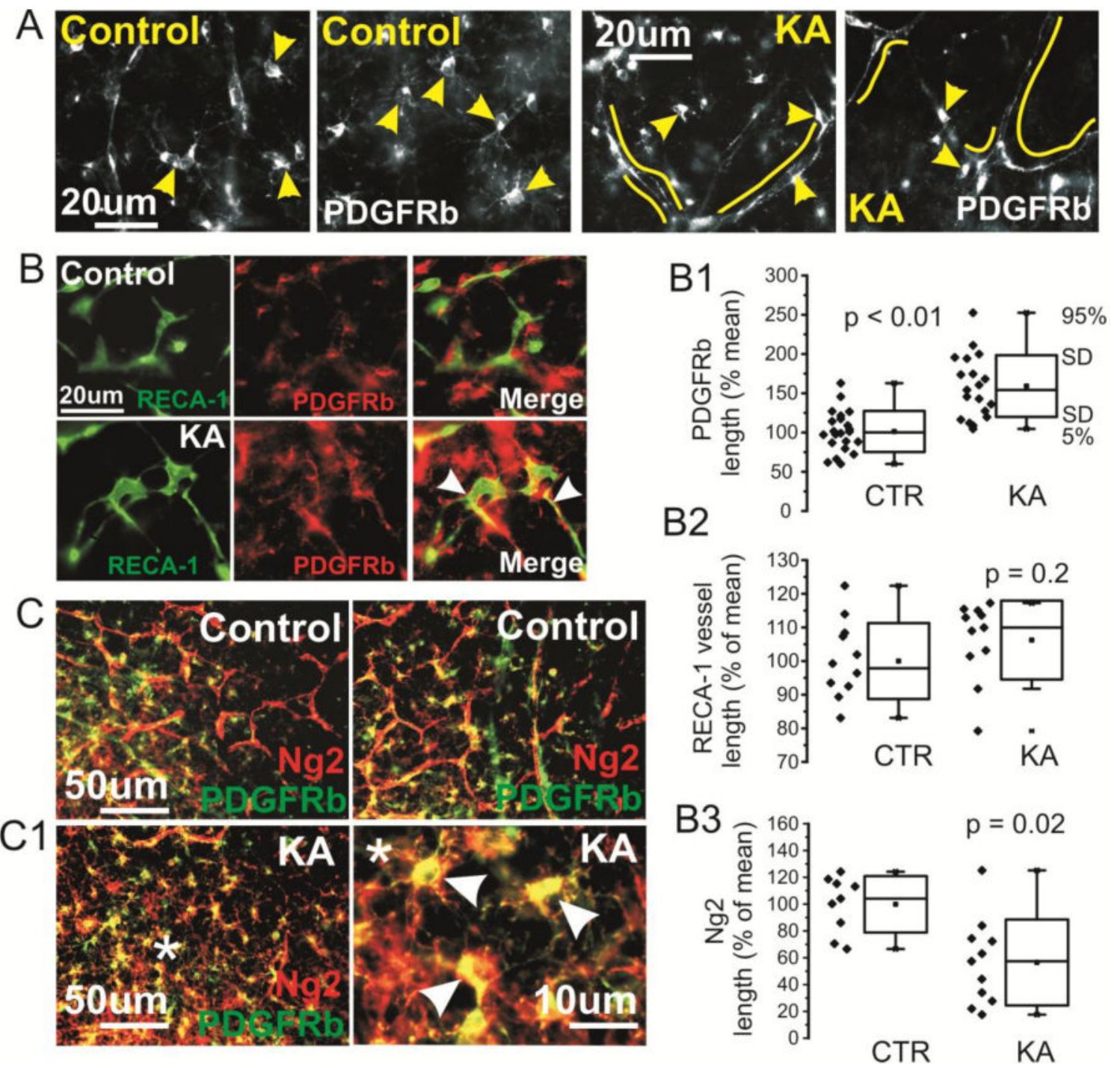

$p=0.02$

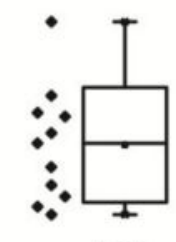

CTR

KA

Figure 5. Organotypic hippocampal culture and PDGFR $\beta$ / NG2 immunoreactivity after induction of seizure-like activity

A) In vitro PDGFR $\beta$ cells distribution shares similarities with the mouse and human tissues (see Figure 6). Parenchymal PDGFR $\beta$ cells were frequent in control slices while vascular rearrangement was also observed after in vitro seizure-like activity (parenchyma arrow heads; vascular continuous yellow line). B) Examples of PDGFR $\beta$ / RECA-1 localization. Note the proximity of PDGFR $\beta$ cells to the RECA-1 positive vasculature in KA (arrow head). B1-B3) Quantification of PDGFR $\beta$ and NG2 lining the vessels and RECA-1 vessel length (see also Methods). Note the PDGFR $\beta$ increase and NG2 decrease. RECA- 1 vessel length did not change significantly after KA treatment. C-C1) NG2 immunoreactivity was prominent at the vasculature in control OHC. Following seizure-like activity (KA) NG2 vascular signal decreased while parenchymal NG2/PDGFR $\beta$ colocalization was observed. 


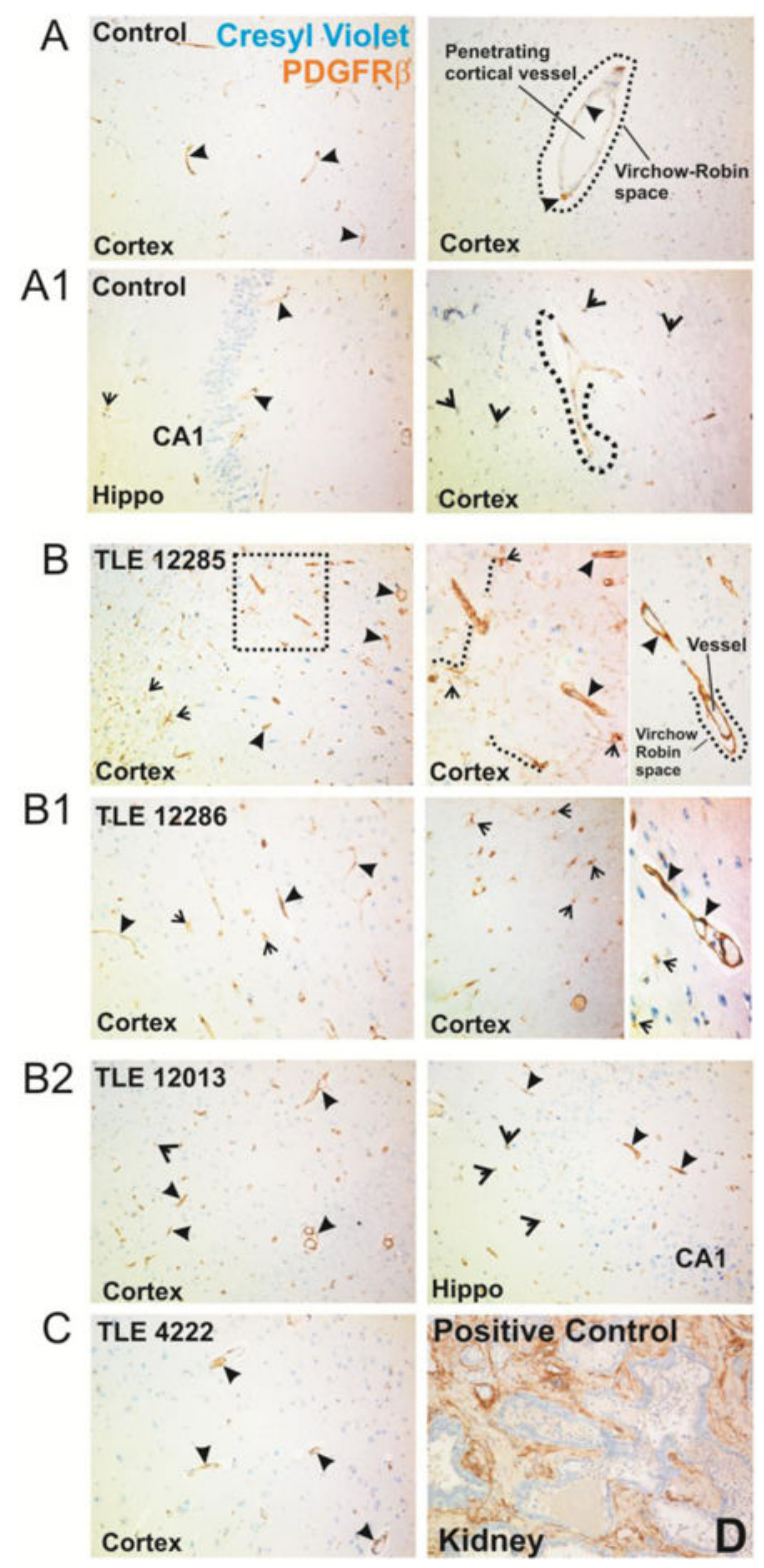

Figure 6. Expression of PDGFR $\beta$ in human TLE brain

A-A1) Control autoptic brain (see Table 1) shows discrete PDGFR $\beta$ expression in the parenchyma (arrows) and in cells associated with the vasculature (arrow heads). B-B2) TLE brains displayed increased PDGFR $\beta$ immune-reactivity. Note the robust staining associated with the vasculature in small caliber and penetrating vessels. A magnification of the dotted panel is provided. In some cases, parenchymal cells branching to the vessels were observed (black dotted line). C) Brain TLE specimen showing modest PDGFRb staining. D) A positive control (kidney) was used to assess Ab specificity. 


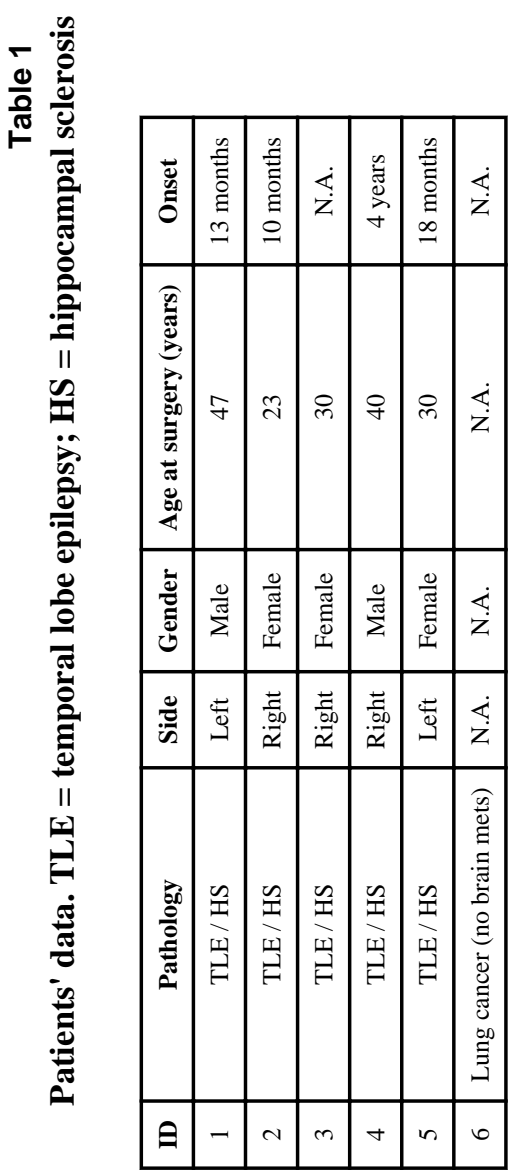

Neurobiol Dis. Author manuscript; available in PMC 2015 November 01. 